\title{
LQR Technique based Second Order Sliding Mode Control for Linear Uncertain Systems
}

\author{
Shital D. Mulje \\ PG Department \\ M.B.E.S College of Engineering, Ambajogai \\ Maharashtra, India
}

\author{
R.M. Nagarale \\ PG Department \\ M.B.E.S College of Engineering, Ambajogai \\ Maharashtra, India
}

\begin{abstract}
In this paper optimal second order sliding mode controller for uncertain systems by using integral sliding surface is proposed. The optimal controller is designed based on linear quadratic regulator (LQR) method for nominal model of the system. In optimal control method, the LQR is combined with sliding mode control to obtain equivalent control and switching control. The sliding mode controller is designed using integral sliding surface to obtain the value of switching control and to equivalent control values are obtained using LQR technique. It is observed that $\mathrm{LQR}$ and integral sliding mode control method are more efficient compare to other conventional techniques. Stabilization of inverted pendulum system is done using the sliding mode control approach. The system of inverted pendulum is used in state space approach. The main advantages of this method is that disturbance rejection, insensitivity to parameter variations and implementation issues are addressed easily using proposed controller stabilization of inverted system. The simulation result conform an advantage of the designed optimal sliding mode control approach in terms of output responses and stabilization of the system.
\end{abstract}

\section{Keywords}

linear uncertain systems, optimal control, linear quadratic regulator, integral sliding surface, non-singular terminal sliding surface, second order sliding mode control.

\section{INTRODUCTION}

The uncertainty in any system can be of two types such as disturbance signals and dynamic perturbations. The former includes input and output disturbance, sensor noise and actuator noise, etc. The latter represents the discrepancy between the mathematical model and the actual dynamics of the system in operation. Uncertainties are unavoidable in a real control system. To overcome such type of problems many researchers have suggested different control methods [12-13]. One of the robust control method was variable structure control based sliding mode control (SMC) has received much attention in the past 60 years for controlling certain as well as uncertain systems [2-6]. In SMC, the system state trajectory was forced to move along a chosen manifold in the state space, called the sliding manifold, by using an appropriate variable structure control signal. The SMC has been widely recognized as a powerful control strategy for its ability of making a control system very robust, which yields complete rejection of external disturbances satisfying the matching conditions. The SMC has widely been extended to incorporate new techniques, such as higher-order sliding mode control, dynamic sliding mode control and optimal sliding mode control.

The optimal control laws (in terms of switch curves and surfaces) were obtained for a variety of second and third-order systems in the early fifties. Then the and constrained cases.
The combination of optimal control with the SMC has given rise to a new field of robust control strategy known as optimal sliding mode control (OSMC). finite and infinite-time linear quadratic optimal control problem has been intensively studied for both the unconstrained The optimal sliding mode control can be designed by combining an integral sliding mode with an optimal controller. Because of integral sliding mode, the order of the system does not get reduce, but system is immune to matched uncertainities once the system trajectories are on sliding manifold. These techniques retain the main advantages of SMC and also yield more accuracy and desired performances. However, OSMC suffers from a problem of chattering. The second order sliding mode controller is developed as a generalization of the first order sliding mode control theory. In second order SMC method, the selected sliding surface has one degree higher with respect to the control. The discontinuous control signal acts on the higher derivatives of sliding surface $(\dot{\boldsymbol{s}}(\mathrm{t}))$ to enforce a sliding motion on $(s=0)$ Indeed, in the second-order sliding mode, the control affects $s$, i.e., the second derivative of the sliding variable. The higher order SMC provides a natural solution to avoid the chattering effect . To overcomes the problem of chattering occurred in SMC, a second order sliding mode controller is proposed .The outline of this paper is as follows. In Section 1, the problem formulation is gives with short description while in Section 2, controller design concepts in view of state space are included. In Subsection 3.1, the optimal control design is included while sliding mode approach with equivalent and switching control is give in Subsection 3.2. The concept of stabilization with inverted pendulum example and conclusions are included in Section 4and5 respectively.

\section{PROBLEM FORMULATION}

The system considered in time domain analysis is given as follows

$$
\dot{x}(t)=(\mathrm{A}+\Delta \mathrm{A}(t)) x(t)+(\mathrm{B}+\Delta \mathrm{B}(t)) u(t)
$$$$
+\zeta(t)
$$

$y(t)=C x(t)$

This is equivalent in differential equation form as $t$ $d x / d t=\mathrm{A} x(t)+\mathrm{B} u(t)+\Delta \mathrm{A} x(t)+\Delta \mathrm{B}(t)+z e t a$

where, ' $\mathrm{A}$ ' is system matrix, $\Delta \mathrm{A}$ is uncertainty (means error in $\mathrm{A}$ matrix ), $\mathrm{B}$ is input matrix, $\Delta \mathrm{B}$ means error or uncertainity in B matrix, this is similar to state space equation given as, $\zeta(t)$ be the exogenous disturbance affecting the system

$d x / d t=\mathrm{A} x+\mathrm{B} u$ 


$$
y=C x+\mathrm{B} u
$$

Which follows assumption in equation (1) Where, $d(t)$ denotes the uncertain part of the system (1). This mean all uncertainty or disturbance is denoted by $d(t)$.

$$
\begin{aligned}
& \dot{x}(t)=\mathrm{A} x(t)+\mathrm{B}(u(t)+d(t)) \\
& y(t)=C x(t)
\end{aligned}
$$

In this paper the $\mathrm{u}$ is designed based on sliding mode control method. The sliding mode controller $\mathrm{u}$ has two controllers as,

$$
u=u_{1}+u_{2}
$$

where , $u_{1}$-equivalent controller and $u_{2}$ - switching controller are discussed in the following section.

\section{CONTROLLER DESIGN}

To design controller $\mathrm{u}$ for the given system, equation (1) can be transformed into controllable canonical form by using linear transformation is obtain as follows,

$$
x(t)=\mathrm{T} z(t)
$$

where $\mathrm{T}$ is the transformation. The state space of the given system can be represented in ideally infinity form. After linear transformation, matrix A can be written as

$$
\begin{aligned}
z(t)= & (\hat{\mathrm{A}}+\Delta \hat{\mathrm{A}}(t)) z(t))+(\quad(\hat{\mathrm{B}}+\Delta \hat{\mathrm{B}}(t)) u(t) \\
& +\hat{\zeta}(t) \\
y(t)= & \hat{C} z(t)
\end{aligned}
$$

where,

$$
\begin{gathered}
\hat{\mathrm{A}}=\mathrm{T}^{-1} \mathrm{AT}=\left[\begin{array}{ccccc}
0 & 1 & 0 & \cdots & 0 \\
0 & 0 & 1 & \cdots & 0 \\
\vdots & \vdots & \vdots & \ddots & \vdots \\
0 & 0 & 0 & \cdots & 1 \\
a_{1} & a_{2} & a_{3} & \cdots & a_{n}
\end{array}\right] \\
\hat{\mathrm{B}}=\mathrm{T}^{-1} \mathrm{~B}=\left[\begin{array}{c}
0 \\
\vdots \\
0 \\
b_{1}
\end{array}\right] \text { and } \hat{C}=C T
\end{gathered}
$$

The parameters

$a_{1}, a_{2}, a_{3}$ are nothing but parameter of transfer function.

$$
e(t)=\left[\begin{array}{c}
e_{1}(t) \\
e_{2}(t) \\
\vdots \\
e_{n}(t)
\end{array}\right]=\left[\begin{array}{c}
z_{1}(t) \\
z_{2}(t) \\
\vdots \\
z_{3}(t)
\end{array}\right]-\left[\begin{array}{c}
z_{d}(t) \\
z_{d}^{1}(t) \\
\vdots \\
z_{d}^{n-1}(t)
\end{array}\right]
$$

$\boldsymbol{e}$ is the error, that is actual state $(z)$ minus desired state ( $z_{d}$ ), The state can be output of the system or speed in case of DC motor. In this paper the error is taken as the state, therefore the equation comes; replace ' $e$ ' by $z$ in equation (7) in paper

$$
\begin{aligned}
& e=z-z_{d} \\
& z=e+z_{d} \\
& \dot{z}=\dot{e}+\dot{z}_{d}
\end{aligned}
$$

$$
\dot{e}(t)=\left[\begin{array}{l}
\dot{e}_{1}(t) \\
\dot{e}_{2}(t) \\
\vdots \\
\dot{e}_{n}(t)
\end{array}\right]=\left(\left[\begin{array}{ccccc}
0 & 1 & 0 & \cdots & 0 \\
0 & 0 & 1 & \cdots & 0 \\
\vdots & \vdots & \vdots & \vdots & \vdots \\
0 & 0 & 0 & \cdots & 1 \\
a_{1} & a_{2} & a_{3} & \cdots & a_{n}
\end{array}\right]\right.
$$

$\left.+\left[\begin{array}{ccccc}0 & 1 & 0 & \cdots & 0 \\ 0 & 0 & 1 & \cdots & 0 \\ \vdots & \vdots & \vdots & \ddots & \vdots \\ 0 & 0 & 0 & \cdots & 1 \\ \Delta a_{1} & \Delta a_{2} & \Delta a_{3} & \cdots & \Delta a_{n}\end{array}\right]\right)$

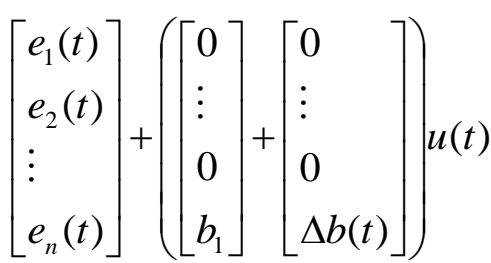

$$
+\left[\begin{array}{c}
0 \\
\vdots \\
0 \\
\zeta_{1}(t)
\end{array}\right]
$$

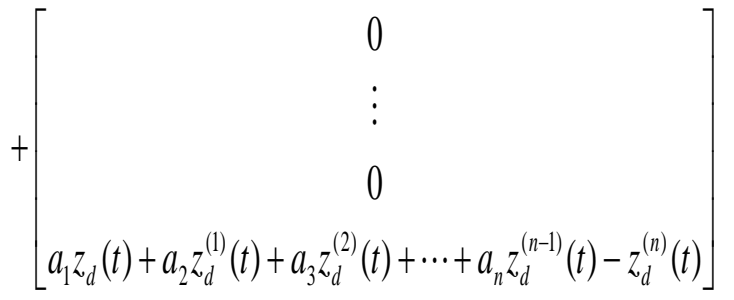


Equation above is nothing but, the following

$$
\begin{aligned}
\dot{e}(t) & =(\hat{\mathrm{A}}+\Delta \hat{\mathrm{A}}(t)) e(t)+(\hat{\mathrm{B}}+\Delta \hat{\mathrm{B}}(t)) u(t) \\
& +\hat{\zeta}(t)+\mathrm{A}_{d}(t)
\end{aligned}
$$

$$
\Delta \hat{\mathrm{A}}(t)=\left[\begin{array}{ccccc}
0 & 1 & 0 & \cdots & 0 \\
0 & 0 & 1 & \cdots & 0 \\
\vdots & \vdots & \vdots & \ddots & \vdots \\
0 & 0 & 0 & \cdots & 1 \\
\Delta a_{1} & \Delta a_{2} & \Delta a_{3} & \cdots & \Delta a_{n}
\end{array}\right]
$$

$\hat{\mathrm{B}}=\left[\begin{array}{c}0 \\ \vdots \\ 0 \\ b_{1}\end{array}\right], \hat{\zeta}(t)=\left[\begin{array}{c}0 \\ \vdots \\ 0 \\ \zeta_{1}(t)\end{array}\right]$

$$
\Delta \hat{\mathrm{B}}=\left[\begin{array}{c}
0 \\
\vdots \\
0 \\
\Delta b(t)
\end{array}\right]
$$

$\mathrm{A}_{d}(t)=\left[\begin{array}{c}0 \\ \vdots \\ 0 \\ a_{1} z_{d}(t)+a_{2} z_{d}^{(1)}(t)+a_{3} z_{d}^{(2)}(t)+\cdots+a_{n} z_{d}^{(n-1)}(t)-z_{d}^{(n)}(t)\end{array}\right]$,

$\Delta \hat{\mathrm{A}}(t) e(t)+\Delta \hat{\mathrm{B}}(t) u(t)+\zeta(t)+\mathrm{A}_{d}(t)$

$=\hat{\mathrm{B}} \psi(t)$

where $\Psi(\mathrm{t})$ is an unknown function

$$
\dot{e}(t)=\hat{\mathrm{A}} e(t)+\hat{\mathrm{B}}\left(u_{1}(t)+u_{2}(t)\right)+\hat{\mathrm{B}} \psi(t)
$$

\subsection{Optimal controller design}

In this section $u_{1}$ can be designed by using LQR system

$$
\dot{e}(t)=\hat{\mathrm{A}} e(t)+\hat{\mathrm{B}}(t) u_{1}(t)
$$

And suppose we want to design state feedback control $\mathrm{u}=\mathrm{K}$ $\mathrm{e}(\mathrm{t})$ to stabilize the system. Design of $\mathrm{K}$ is a tradeoff between the transient response and control effort. The optimal control approach to this design trade off is to define the performance index (cost function) $J=\int_{0}^{\infty}\left[e^{\tau} Q e(\tau)+u_{1}(\tau)^{\mathrm{T}} \mathrm{R}\right.$ $\left.u_{1}(\tau)\right] \mathrm{d} \tau$
$\mathrm{Q} \in R^{n \times n}$ is positive semi-definite and $R \in R$ is positive definite weighing matrix. T he optimal control law is $u_{1}(t)$ obtained as

$$
u_{1}(t)=-R^{-1} \hat{B} \operatorname{Pe}(t)=-K e(t)
$$

Where $K=R^{-1} \hat{\mathrm{B}}^{\mathrm{T}} P$ and $p$ is symmetric, positive definite matrix .

The nominal system equation (12) is stabilized by the optimal control $u_{1}(t)$ which is obtained by minimizing the performance index from equation (13).

In this stabilization problem, it can be considered that the desired trajectory $x_{d}(t)=0$. Hence tracking error $e(t)$ can be describe as

$$
e(t)=\left[\begin{array}{l}
e_{1}(t) \\
e_{2}(t) \\
e_{3}(t) \\
e_{4}(t)
\end{array}\right]=\left[\begin{array}{l}
z_{1}(t) \\
z_{2}(t) \\
z_{3}(t) \\
z_{4}(t)
\end{array}\right]
$$

In this paper given value of $\mathrm{Q}$ and $\mathrm{R}$ to minimize the control input, the performance index $\mathrm{J}$ is given by From equation (14) similarly $e(\tau)=z(\tau)$

The feedback control $u_{1}(t)$ is calculated by using LQR technique.

\subsection{Sliding mode controller design}

$$
s(t)=G\left[e(t)-e_{0}-\int_{0}^{\tau} \dot{\varphi}(\tau) d \tau\right] d \tau
$$

where $\mathrm{G}$ is design parameter, $e_{0}$ be the error condition

$$
\dot{\varphi}(t)=\hat{\mathrm{A}} e(t)+\hat{\mathrm{B}} u_{1}(t)
$$

Differentiation Equation (16) with respective time

$$
\dot{s}(t)=G[\dot{e}(t)-\dot{\varphi}(t)]
$$

Equation (12) and equation (17) put in equation (18)

$$
\begin{aligned}
& \dot{s}(t)=\hat{\mathrm{A}} e(t)+\hat{\mathrm{B}}(t) u_{1}(t)-\hat{\mathrm{A}} e(t)-\hat{\mathrm{B}}(t) u_{1}(t) \\
& \dot{s}(t)=0
\end{aligned}
$$

In this method $u_{1}(t)$ is not calculated from equation (19).So $\mathrm{LQR}$ technique is used Equation (11) and equation (17) put in equation (18)

$\dot{s}(t)=G\left[\hat{\mathrm{A}} e(t)+\hat{\mathrm{B}}\left(u_{1}(t)+u_{2}(t)\right)+\hat{\mathrm{B}} \psi(t)-\right.$ $\left.\hat{\mathrm{A}} e(t)-\hat{\mathrm{B}}(t) u_{1}(t)\right]$

$\dot{s}(t)=\left[G \hat{\mathrm{B}} u_{2}(t)+\hat{\mathrm{B}} \psi(t)\right]$

We get $u_{2}(t)$ in equation (20) 
Above equation reaching phase is eliminated in the integral sliding mode control switching control is designed based on $\eta$ - reachability condition is given as

$\dot{s}(t)<-\rho \operatorname{sgn}(s(t))$

Where $\rho>0$ and

$\operatorname{sgn}(s(t))= \begin{cases}1 & s(t)>0 \\ -1 & s(t)<0 \\ 0 & s(t)=0\end{cases}$

From equation (22) and (21) is obtained by

$G \hat{\mathrm{B}} u_{2}(t)<[\rho \operatorname{sgn}(s(t))+\hat{\mathrm{B}} \psi(t)]$

$u_{2}(t)<-\left((G \hat{\mathrm{B}})^{-1}[\rho \operatorname{sgn}(s(t))\right.$

$+G \hat{\mathrm{B}} \psi(t)]$

Where, $G \hat{\mathrm{B}}$ Invertible means the matrix is has inverse. In this sgn means signum function given in From equation (24) it is clear that the switching control $u_{2}(t)$ is effected by sign function of the sliding mode. This sign function is created chattering in the control input. ISMC, the chattering effect is eliminated, the proposes second order sliding mode manifold.

Design of equation(16) is not required for knowledge of initial condition .But the very beginning of sliding surface is not system. Hence a non-singular terminal sliding surface is added to guarantee finite time convergence of $s(t)$. So nonsingular terminal sliding surface $\sigma(t)$ can be designed by

$\sigma(t)=s(\mathrm{t})+\delta \dot{s}(t)^{\alpha / \beta}$

Where $\delta>0$ is switching gain

and $\alpha, \beta$ are the value of variable structure system with sliding mode control .In this system is given condition.

$\alpha, \beta \in[2 n+1: n$ is an integer $]$

Alpha and beta belongs to $[2 n+1: n]$ means the value of alpha and beta is between : $n$ to $2 n+1$. Where ' $n$ ' is any integer value. Example if $n=1$, alpha and beta are having values in between 1 and 3

and $1<\frac{\alpha}{\beta}<2$

Equation (28) is another condition on alpha and beta, the ratio of alpha/beta should be between 1 and 2 .

The linear sliding manifold $\mathrm{s}(\mathrm{t})$ in equation (16) and nonsingular terminal sliding manifold $\sigma(\mathrm{t})$ is combined to realize the second order SMC by designed the constant plus proportion reaching law [15] gives rise to $\dot{\sigma}(t)=-\eta_{1} \operatorname{sgn}(\sigma(t))-\varepsilon_{1} \sigma(t)$

Where $\eta_{1}>0$ and $\varepsilon_{1}>0$.

Differentiation equation (25) with respective to $t$

$$
\begin{aligned}
& \dot{\sigma}(t)=\dot{s}(t)+\delta \frac{\alpha}{\beta} \dot{s}(t)^{\alpha / \beta-1} \ddot{s}(t) \\
& =\delta \frac{\alpha}{\beta} \dot{s}(t)^{\alpha / \beta-1}\left(\frac{\beta}{\delta \alpha} \dot{s}(t)^{2-\alpha / \beta}+\ddot{s}(t)\right)
\end{aligned}
$$

For the parameter $\alpha, \beta$ equation (27) and (28) value is given by

$\dot{s}(t)^{\alpha / \beta-1}>0$ for $\dot{s}(t) \neq 0$

$\dot{s}(t)^{\alpha / \beta-1}=0$ for $\dot{s}(t)=0$

From equation (26), (27) and (31). The term

$\delta(\alpha / \beta) \dot{s}(t)^{\alpha / \beta-1}$ (30) can be substituted by a scalar $\eta_{1}>0$ for $\dot{s}(t) \neq 0$ in this condition is given by

$\dot{\sigma}(t)=\eta_{2}\left(\frac{\beta}{\delta \alpha} \dot{s}(t)^{2-\alpha / \beta}+\ddot{s}(t)\right)$

Substituting the value of $\dot{\sigma}(t)$ from (29), (32) can be expressed as

$=\eta_{2}\left(\frac{\beta}{\delta \alpha} \dot{s}(t)^{2-\alpha / \beta}+\ddot{s}(t)\right)=-\eta_{1} \operatorname{sgn}(\sigma(t))-\varepsilon_{1} \sigma(t)$

$\frac{\beta}{\delta \alpha} \dot{s}(t)^{2-\alpha / \beta}+\ddot{s}(t)=-\eta_{1} \operatorname{sgn}(\sigma(t))-\varepsilon_{1} \sigma(t)$

(33) Where $\eta=\eta_{1} / \eta_{2}>0$ and $\varepsilon=\varepsilon_{1} / \varepsilon_{2}>0$.

Then (33) can be rewritten as

$$
\begin{array}{r}
\ddot{s}(t)=-\eta \operatorname{sgn}(\sigma(t))-s \sigma(t) \\
-\frac{\beta}{\delta \alpha} \sigma(t) \dot{s}(t)^{2-\alpha / \beta}
\end{array}
$$

(34) Differentiating (20) given by

$$
\ddot{s}(t)=G\left[\hat{\mathrm{B}} \dot{u}_{2}(t)+\hat{\mathrm{B}} \psi(t)\right]
$$

(35) From Equation(34) and(35), the switching control law

$$
\begin{aligned}
& u_{2}(t)=-\int_{0}^{t}(G \hat{\mathrm{B}})^{-1}\left[\frac{\beta}{\delta \alpha} \sigma(\tau) \dot{s}(\tau)^{2-\alpha / \beta}\right. \\
& +\eta \operatorname{sgn} \sigma(t)+\varepsilon \sigma(\tau)] d \tau
\end{aligned}
$$

Where the design parameters are chosen in 
Such a way that $\eta$ and $\varepsilon$

$|G \hat{\mathrm{B}} \dot{\psi}(t)|<\eta \quad$ (From equation 35)

\section{SIMULATION AND RESULT}

Consider example of inverted pendulum, Since the analysis (state space model) and control design techniques that I will employ in this problem apply only to linear systems, these equations need to be linearized. Specifically, I will linearize the equations about the vertically upward equilibrium position, $\theta=0$, and will assume that the system stays within a small neighborhood of this equilibrium

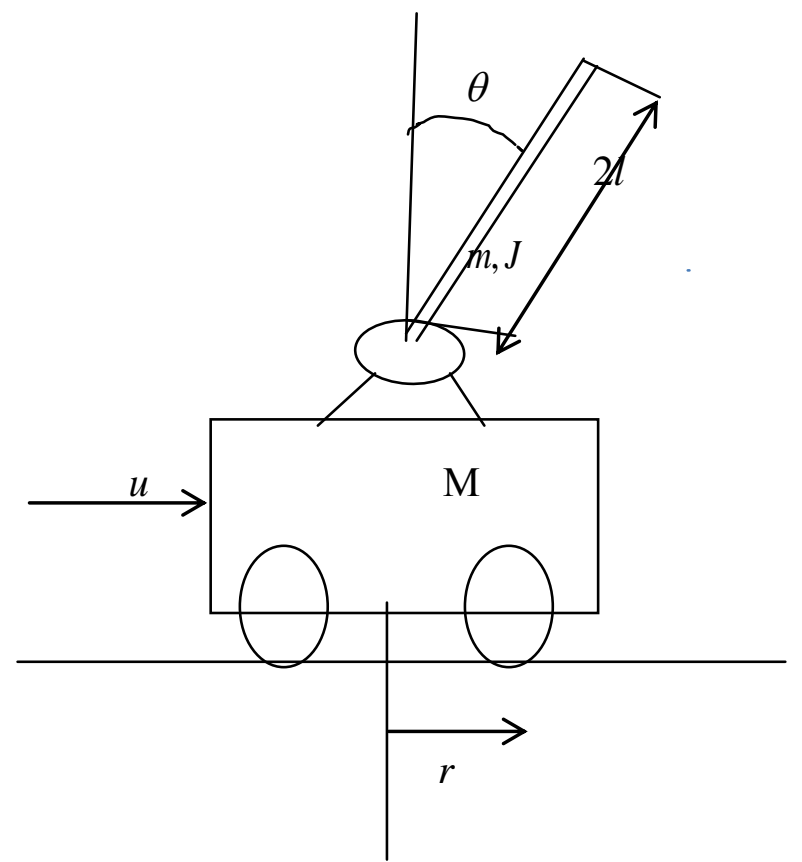

Fig 1.inverted pendulum

As shown in fig(1) cart having mass $\mathrm{M}$ is displaced by an amount of the $r$ due to external force $u$. M be the mass of pendulum mounted on cart has an angular displacement $\theta .2 l$ be the length of the pendulum and $J$ be the inertia of pendulum.

Inverted pendulum is given by

$$
\begin{aligned}
& {\left[\begin{array}{l}
\dot{x}_{1}(t) \\
\dot{x}_{2}(t) \\
\dot{x}_{3}(t) \\
\dot{x}_{4}(t)
\end{array}\right]=\left[\begin{array}{cccc}
0 & 0 & 1 & 0 \\
0 & 0 & 0 & 1 \\
0 & -1 \cdot 933 & -1 \cdot 987 & 0 \cdot 009 \\
0 & 36 \cdot 977 & 6 \cdot 258 & -0 \cdot 173
\end{array}\right]} \\
& {\left[\begin{array}{l}
x_{1}(t) \\
x_{2}(t) \\
x_{3}(t) \\
x_{4}(t)
\end{array}\right]+\left[\begin{array}{l}
0 \\
0 \\
0 \cdot 320 \\
-1 \cdot 009
\end{array}\right](u(t)+d(t))}
\end{aligned}
$$

Where, states $x_{1}(t), x_{2}(t), x_{3}(t), x_{4}(t)$ be the cart displacement of the pendulum $\mathrm{r}(\mathrm{t})$, angular displacement of the pendulum $\dot{\theta}(\mathrm{t}) \cdot \mathrm{d}(\mathrm{t})$ is the disturbance, value of $\mathrm{d}(\mathrm{t})$ is given by $1.5 \sin (\pi t / 3)+\cos t$. Equation (39) is to be stabilize with control input similarly transformation is used to

$$
z(t)=\left[\begin{array}{cccc}
-0.1010 & -0.0321 & -0.0005 & -0.0002 \\
0 & -0.0047 & -0.1010 & -0.0321 \\
0 & -0.9906 & 0 & 0 \\
0 & 0.0001 & 0 & -0.9906
\end{array}\right]
$$

$$
x(t)
$$

(37)Equation (36) is obtained in the controllable canonical form a

$$
\dot{z}(t)=\left[\begin{array}{cccc}
0 & 1 & 0 & 0 \\
0 & 0 & 1 & 0 \\
0 & 61.38 & 36.68 & -2.16
\end{array}\right] z(t)+\left[\begin{array}{l}
0 \\
0 \\
0 \\
1
\end{array}\right]
$$

$(u(t)+d(t))$

In this paper design parameters of optimal second order sliding mode controller is given by

a) $\hat{\mathrm{B}}=\left[\begin{array}{lll}0 & 0 & 1\end{array}\right]^{\mathrm{T}}, \mathrm{G}$ is chosen in such that $G \hat{\mathrm{B}}$ is invertible. Accordingly , $G=\left[\begin{array}{llll}0 & 0 & 0 & 1\end{array}\right]$

b) from equation(27) and equation(28) is given by value of

$$
\alpha=7, \beta=5
$$

c) terminal sliding surface to be stable,$\delta$ should be positive value $\delta=0 \cdot 15$

d) $\eta, \varepsilon$ be the design parameter of the switching control law $\eta=3, \varepsilon=0.1$

1. The following graphs are of inverted pendulum by applying the proposed OSOSMC \& LQR system.

2. $d(t)$ is the disturbance, value of $d(t)$ is given by $1.5 \sin (\pi t / 3)+\cos t$. These value put in program to obtain similar graph because SMC is the eliminate the disturbance

3. I have shown SMC without disturbance \& SMC with disturbance 


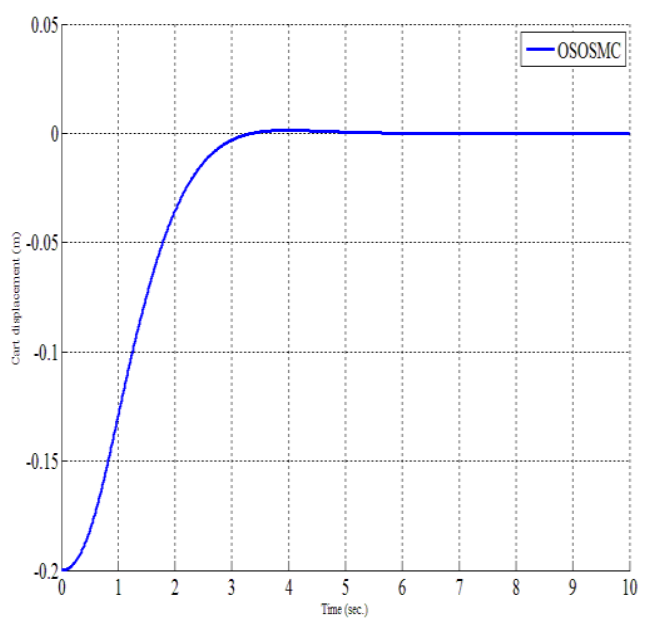

Fig 2.State $x_{1}(t)$

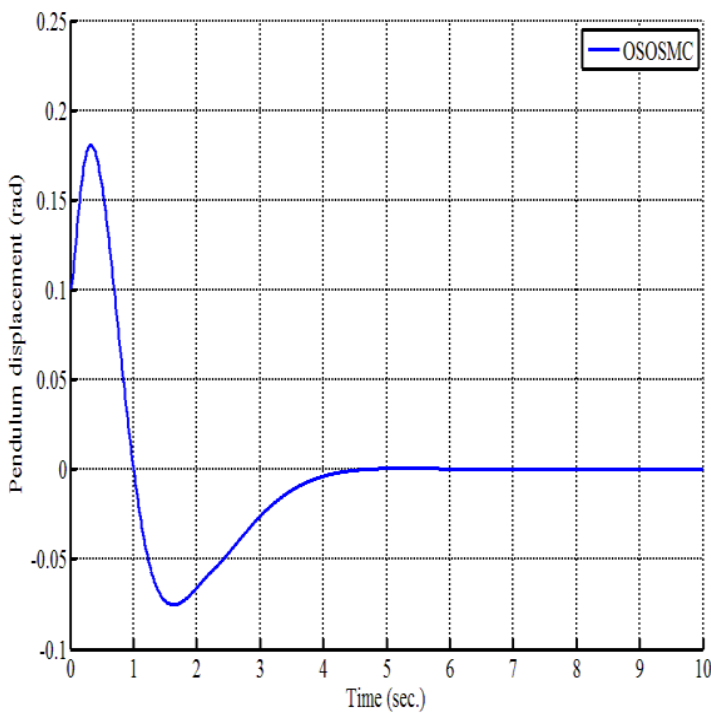

Fig 3 .State $x_{2}(t)$

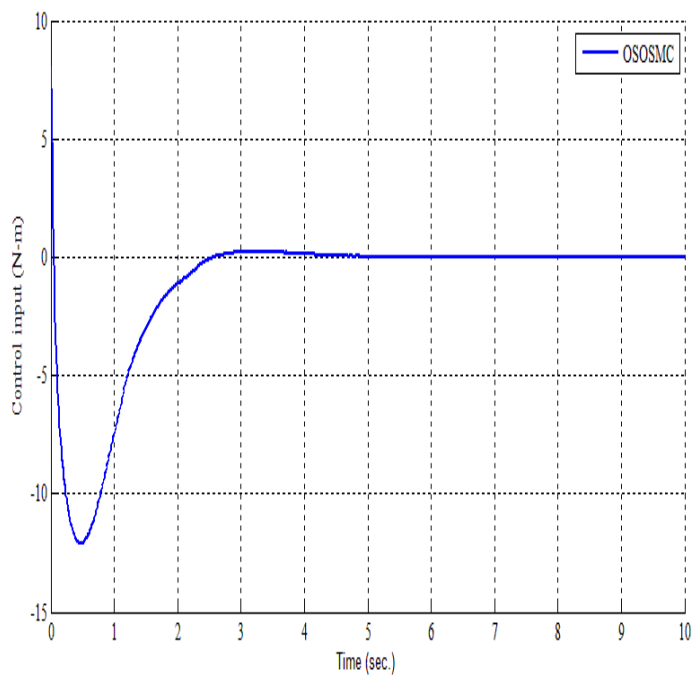

Fig 4 Control input

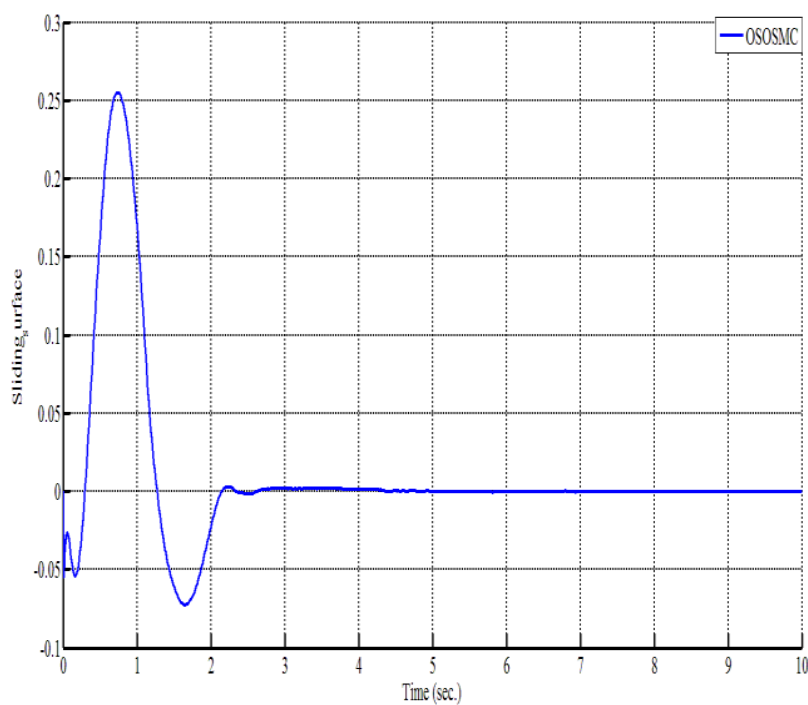

Fig 5. Sliding Surface

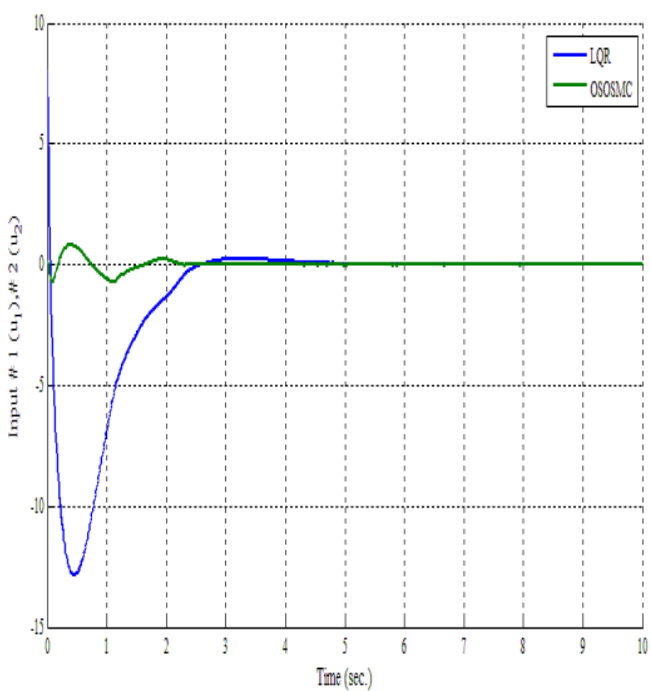

Fig 6 . equivalent control $u_{1}$ by using $\mathrm{LQR}$ and switching control $u_{2}$ by using OSOSMC

\section{CONCLUSION}

This paper proposed optimal second order sliding mode control for uncertain system by using integral sliding surface. The stabilization of inverted pendulum system is made using the integral sliding surface designed based SMC. The result describes the design of integral sliding surface designed based SMC to obtain value of switching control which is used to control the hard system such as inverted pendulum . Linear quadratic regulator is used effectively to obtain value of equivalent control. It is observed that LQR and ISMC method are more efficient to control the position of the pendulum at predefined position. The main advantages of the proposed method includes the disturbance rejection as well as insensitivity to parameter variations. The simulation result shows an effectiveness of the proposed optimal sliding mode control approach in terms of output responses and stabilization of the system. 


\section{REFERENCES}

[1] Madhulika Das , Chitralekha Mahanta" Optimal second order sliding mode control for linear uncertain systems," ISA. Trans proceeding sliding mode control.

[2] I. J. Nagrath, M.Gopal control system Engg. International publication fifth edition

[3] Utkin V. "Sliding modes in control and optimization”, Berlin: Springer-Verlag; 1992.

[4] Utkin V. "Variable structure systems with sliding modes", .IEEE Trans Autom Control 1977;22(2):212 22 .

[5] XuR,Özgüner Ü. "Optimal sliding mode control for linear systems", .In :IEEE proceedings on variable structure systems.2006.p.143-8.

[6] young KD,Özgüner Ü. "Sliding- mode design for robust linear optimal control”,Automatica 1997;33(7):1313-23.

[7] Laghrouche S, Plestan F, Glumineau A. Higher order sliding mode control based on integral sliding mode".Automatica2007;43(3):531-7.
[8] Feng Y,Han X, Wang Y ,Yu X. Second order terminal sliding mode control of uncertain multivariablesystems.IntJControl2007;80(6):856-62.

[9] Hung J, Gao W, Hung J .Variable structure control :a survey. IEEE Trans Ind Electron 1993;40(1):2-22.

[10] Pang H-P, Yang Q. Optimal sliding mode output tracking control for linear systems with uncertainties.In:2010international conference on machine learning and cybernetics (ICMLC), vol.2,2010.p.942-6.

[11] Dong R, Gao H-W ,Pan Q-X .Optimal sliding mode control for nonlinear systems with uncertainties In:IEEEproceedingsoncontrolanddecision,2011.p. 2098-103.

[12] Schmitendorf W. Barmish B. Robust asymptotic tracking for linear systems with unknown parameters .Automatica1986;22(3):355-60

[13] Marino R, Tomei P.Global adaptive output-feedback control of nonlinear systems, Part i: linear parameterization. IEEE Trans Automatic Control1993;38(1): 17-32. 